\title{
Dual-Functional PLGA Nanoparticles Co-Loaded with Indocyanine Green and Resiquimod for Prostate Cancer Treatment
}

This article was published in the following Dove Press journal: International Journal of Nanomedicine

\author{
Wenfeng Lin ${ }^{1, *}$ \\ Chaoming $\mathrm{Li} \mathbb{( D D}^{1,2, *}$ \\ Naijin $\mathrm{Xu}^{1,3}$ \\ Masami Watanabe ${ }^{1,4}$ \\ Ruizhi Xue' \\ Abai $X u^{2}$ \\ Motoo Araki (iD) \\ Ruifen Sun ${ }^{5}$ \\ Chunxiao Liu $^{2}$ \\ Yasutomo Nasu' \\ Peng Huang $\mathbb{D}^{1,2,6}$ \\ 'Department of Urology, Okayama \\ University Graduate School of Medicine, \\ Dentistry and Pharmaceutical Sciences, \\ Okayama, Japan; ${ }^{2}$ Department of \\ Urology, Zhujiang Hospital, Southern \\ Medical University, Guangzhou, People's \\ Republic of China; ${ }^{3}$ Department of \\ Urology, Sir Run Run Shaw Hospital, \\ Zhejiang University School of Medicine, \\ Hangzhou, People's Republic of China; \\ ${ }^{4}$ Center for Innovative Clinical Medicine, \\ Okayama University Hospital, Okayama, \\ Japan; ${ }^{5}$ Center for Scientific Research, \\ Yunnan University of Chinese Traditional \\ Medicine, Kunming, People's Republic of \\ China; ${ }^{6}$ Okayama Medical Innovation \\ Center, Okayama University, Okayama, \\ Japan
}

*These authors contributed equally to this work

Correspondence: Peng Huang Department of Urology, Okayama University Graduate School of Medicine, Dentistry and Pharmaceutical Sciences,

Okayama, 700-8558, Japan

$\mathrm{Tel}+8 \mathrm{I}-86-235-7997$

Fax +8I-86-235-7884

Email huangpeng509@gmail.com
Purpose: With the advance of screening techniques, there is a growing number of low-risk or intermediate-risk prostate cancer $(\mathrm{PCa})$ cases, remaining a serious threat to men's health. To obtain better efficacy, a growing interest has been attracted to develop such emerging treatments as immunotherapy and focal therapy. However, few studies offer guidance on whether and how to combine these modalities against PCa. This study was designed to develop dual-functional nanoparticles (NPs) which combined photothermal therapy (PTT) with immunotherapy and determine the anti-tumor efficacy for PCa treatment.

Methods: By a double emulsion technique, the drug nanocarrier, poly(lactic-co-glycolic acid) or PLGA, was applied for co-loading of a fluorescent dye, indocyanine green (ICG) and a toll-like receptor 7/8 (TLR7/8) agonist resiquimod (R848) to synthesize PLGA-ICG-R848 NPs. Next, we determined their characteristic features and evaluated whether they inhibited the cell viability in multiple PCa cell lines. After treatment with PLGA-ICG-R848, the maturation markers of bone marrow-derived dendritic cells (BMDCs) were detected by flow cytometry. By establishing a subcutaneous xenograft model of mouse PCa, we explored both the anti-tumor effect and immune response following the NPs-based laser ablation.

Results: With a mean diameter of $157.7 \mathrm{~nm}$, PLGA-ICG-R848 exhibited no cytotoxic effect in PCa cells, but they significantly decreased RM9 cell viability to $(3.9 \pm 1.0) \%$ after laser irradiation. Moreover, PLGA-ICG-R848 promoted BMDCs maturation with the significantly elevated proportions of CD11c $+\mathrm{CD} 86+$ and $\mathrm{CD} 11 \mathrm{c}+\mathrm{CD} 80+$ cells. Following PLGA-ICGR848-based laser ablation in vivo, the decreased bioluminescent signals indicated a significant inhibition of PCa growth, while the ratio of splenic natural killer (NK) cells in PLGA-ICG-R848 was $(3.96 \pm 1.88) \%$ compared with $(0.99 \pm 0.10) \%$ in PBS group, revealing the enhanced immune response against PCa.

Conclusion: The dual-functional PLGA-ICG-R848 NPs under laser irradiation exhibit the anti-tumor efficacy for PCa treatment by combining PTT with immunotherapy.

Keywords: prostate cancer, PLGA, indocyanine green, resiquimod, photothermal therapy, immunotherapy

\section{Introduction}

Prostate cancer ( $\mathrm{PCa}$ ) poses a serious threat to men's health with the second highest incidence of all malignant tumors in men worldwide. ${ }^{1}$ According to the Cancer Statistics 2020, it has been estimated that there would be 191,930 new cases of PCa, accounting for $21 \%$ of all male malignancies, and that 33,330 patients would die of this disease in the United States. ${ }^{2}$ Therapeutic strategies for PCa consist of 
surveillance, localized therapy and systemic therapy. Localized therapy includes surgery, radiation therapy and focal therapy while systemic therapy includes hormonal therapy, chemotherapy and immunotherapy. ${ }^{3,4}$ Generally, treatment options depend partly on whether $\mathrm{PCa}$ is localized, high-risk, advanced or recurrent. Due to the limited efficacy and side effects of monotherapy, effective combination therapies or novel treatment regimens still need to be developed to obtain better efficacy outcomes. ${ }^{5}$ Recently, a growing interest has been attracted to such novel treatments as immunotherapy ${ }^{6}$ and PTT therapy. ${ }^{7}$ However, few available studies can offer guidance to whether and how to combine these modalities, and their synergistic applications may give another perspective in the treatment of PCa.

Accumulating evidence suggests that human PCa does not respond well to mono-immunotherapy due to the low infiltration of effective immune cell types, which is also known as immunologically "cold". ${ }^{8}$ Cancer vaccines are considered as promising strategies to modify the tumor microenvironment (TME) from "cold" to "hot" type." Relative to conventional cancer vaccines, in situ cancer vaccines and effective adjuvants recently show great potential to overcome the limitations of poor immunogenicity, high cost and safety concerns. ${ }^{10}$ In situ vaccination aims to produce tumor-associated antigens (TAAs) at the tumor site without prior identification and isolation, which will further trigger a TAA-specific adaptive immune response. ${ }^{11}$ The application of immune adjuvants can effectively improve the immunogenicity of vaccines and enhance the specific immune response. Based on smallmolecule agonists at toll-like receptor 7/8 (TLR7/8), a growing number of clinical trials have been conducted to assess the anti-tumor immunity in multiple cancers. ${ }^{12}$ As a TLR7/8 agonist approved by Food and Drug Administration (FDA), resiquimod (R848) may be an immune adjuvant or a single immunomodulatory drug with important application prospects in cancer treatment, but very few studies have been conducted on the potential impacts of R848 used to treat PCa. It must be noted that free $\mathrm{R} 848$ rapidly induces a systemic production of inflammatory cytokines, leading to potential adverse events and safety risks, ${ }^{13}$ which can be reduced by its encapsulation in NPs.

The emerging treatment modality, focal therapy, mainly consists of cryotherapy, high-intensity focused ultrasound (HIFU), laser ablation, photodynamic therapy (PDT), irreversible electroporation, radiofrequency ablation and focal brachytherapy. ${ }^{14}$ With Prostate Specific Antigen (PSA) screening being widely used, an increasing number of $\mathrm{PCa}$ cases are diagnosed as low-risk or intermediate-risk. ${ }^{15}$ Therefore, focal therapy will play a more critical role due to both the ablation of prostate cancer lesions similar to surgical removal and the protective effects of urinary and sexual functions. ${ }^{16}$ However, further clinical and basic research data are still needed to confirm its therapeutic effects. As an amphiphilic nearinfrared (NIR) fluorescent dye, indocyanine green (ICG) can not only efficiently convert the absorbed light energy to heat for photothermal therapy (PTT) but also induce reactive oxygen species (ROS) production for $\mathrm{PDT},{ }^{17}$ which may serve as a promising anticancer reagent by laser ablation. However, it should be noted that both the instability of ICG in water, light, heat and the rapid clearance in vivo pose serious obstacles to its practical use. Increasing evidence suggests that ICG encapsulated in nanoparticles (NPs) can overcome the above deficiencies and exhibit the enhanced effects of cancer therapy. ${ }^{18}$

As one of the most widely applied drug nanocarriers, poly(lactic-co-glycolic acid) or PLGA exhibits the favorable properties such as sustained drug release, biocompatibility, non-toxicity, non-immunogenicity and biodegradability. ${ }^{19,20}$ The present research aimed to develop a dual-functional PLGA NPs co-loaded with ICG and R848 (PLGA-ICG-R848 NPs), and then determined their in vitro and in vivo anticancer efficacy for $\mathrm{PCa}$ treatment when irradiated with $808 \mathrm{~nm}$ laser. Our findings could give another perspective in the development of potential therapeutics against PCa by combining PTT with immunotherapy.

\section{Materials and Methods Materials and Reagents}

Poly(lactic-co-glycolic acid) (PLGA, \#719889), resiqui$\bmod (\mathrm{R} 848, \#$ SML0196) and indocyanine green (ICG, \#I2633) were purchased from Sigma-Aldrich. Dichloromethane (DCM, \#270997), dimethyl sulfoxide (DMSO, \#472301) and polyvinyl alcohol (PVA, \#363170) were also provided by Sigma-Aldrich. Recombinant mouse GM-CSF (BioLegend, \#576304), recombinant mouse IL-4 (BioLegend, \#574304), lipopolysaccharides (LPS, Sigma-Aldrich, L4391), 1X RBC Lysis Buffer (Invitrogen, \#00-4333) and 100-mm ultra-low attachment culture dish $\left(\right.$ Corning $\left.{ }^{\circledR}, \# 3262\right)$ were obtained for BMDCs culture. 


\section{Cell Lines and Cell Culture}

The human prostate cancer cell lines PC-3, LNCaP and DU-145 were purchased from American Type Culture Collection (ATCC). The mouse prostate cancer cell line RM9 was a gift from Professor T.C. Thompson of University of Texas, USA. RM9 and DU-145 cells were cultured in Dulbecco's modified eagle medium (DMEM; Gibco, Invitrogen, USA) supplemented with 10\% fetal bovine serum (FBS; Gibco, Invitrogen, USA), 1\% penicillin-streptomycin (Gibco, Invitrogen, USA), and maintained at $37^{\circ} \mathrm{C}$ in a humidified atmosphere containing $5 \%$ $\mathrm{CO}_{2}$. PC-3 and LNCaP cells were cultured in the same environment, but in F-12 and RMPI-1640 media, respectively, containing $10 \%$ FBS and $1 \%$ penicillinstreptomycin.

\section{Preparation of PLGA-ICG-R848 NPs}

PLGA-ICG-R848 was synthesized using a double emulsion/solvent evaporation technique. ${ }^{21}$ Briefly, $60 \mathrm{mg}$ PLGA was co-dissolved in $2 \mathrm{~mL}$ DCM with $0.8 \mathrm{mg}$ R848. Then, $0.2 \mathrm{~mL}$ of $3 \mathrm{mg} / \mathrm{mL}$ ICG solution was added to the mixture above, which was emulsified for 2 min using ultrasonicator (Bioruptor UCD-250, Diagenode, Belgium). The primary emulsion was next ultrasonicated with $5 \mathrm{~mL}$ of $2.5 \%(\mathrm{w} / \mathrm{v})$ PVA aqueous solution for $6 \mathrm{~min}$ to produce the $\mathrm{w} / \mathrm{o} / \mathrm{w}$ double emulsion. Transferred the w/ $\mathrm{o} / \mathrm{w}$ emulsion to $5 \mathrm{~mL}$ of $0.25 \%(\mathrm{w} / \mathrm{v})$ PVA aqueous solution and facilitated organic solvent evaporation under magnetic stirring for $4 \mathrm{~h}$. The prepared PLGA-ICG-R848 NPs were collected by centrifugation at 23,000 g for 20 min, and washed twice with Milli-Q water. Finally, the freeze-dried powder of PLGA-ICG-R848 NPs was obtained after lyophilized for $24 \mathrm{~h}$ and stored at $4^{\circ} \mathrm{C}$ in the dark before use.

\section{Characterization of PLGA-ICG-R848 NPs} The PLGA-ICG-R848 NPs were firstly dispersed in Milli-Q water and then presented to a dynamic light scattering (DLS) device named Zetasizer Nano ZSP (Malvern Panalytical, UK) for the determination of their particle sizes, polydispersity index (PDI) and zeta potential. The morphology and surface structure of NPs were characterized by scanning electron microscope (SEM; S-4800, Hitachi, Japan). The UV-VIS-NIR absorption spectra of free ICG, PLGA-ICG and PLGA-ICG-R848 was confirmed by a microvolume spectrophotometer (DeNovix Inc., DS-11, USA). The FlexStation ${ }^{\circledR} 3$ Multi-
Mode Microplate Reader (Molecular Devices, USA) was used to measure the concentration of ICG with absorbance at $780 \mathrm{~nm}$ wavelength. High performance liquid chromatography (HPLC, LC-20AT, Shimadzu, Japan) coupled with an ultraviolet-visible detector (SPD-20A, Shimadzu, Japan), together with XTerra RP18 column (3.0 $\mathrm{mm} \times 150 \mathrm{~mm}, 5.0 \mu \mathrm{m}$, Waters, USA) were applied to detect the concentration of $\mathrm{R} 848$ at $250 \mathrm{~nm}$ (Supplementary Figure 1A). HPLC analysis was performed using mobile phase A $(0.1 \%$ trifluoroacetic acid in acetonitrile) and mobile phase $\mathrm{B}(0.1 \%$ trifluoroacetic acid in water). The standard curve and linear regression equation of R848 are shown in Supplementary Figure 1B and $\underline{\mathrm{C}}$. For ICG measurement, we firstly collected the supernatant after centrifugation, and determined the concentration of free ICG in supernatant. Then, we calculated the weight of entrapped ICG by subtracting the weight of free ICG in supernatant from overall feeding weight. Encapsulation efficiency (EE\%) and loading capacity $(\mathrm{LC} \%)$ were calculated using the following formulation:

$$
\begin{aligned}
& E E \%=\frac{\text { Weight of entrapped drug }}{\text { Over all feeding weight }} \times 100 \% \\
& L C \%=\frac{\text { Weight of entrapped drug }}{\text { Weight of NPS }} \times 100 \%
\end{aligned}
$$

\section{Cellular Uptake Experiments}

RM9 cells were seeded on the coverslips in 24-well plates at a density of $3 \times 10^{4}$ cells per well and allowed to adhere overnight. Then the cells were treated for $2 \mathrm{~h}$ or $12 \mathrm{~h}$ with PLGA, PLGA-ICG, PLGA-R848 and PLGA-ICG-R848 at the same concentration of ICG $(40 \mu \mathrm{M})$ or R848 $(6.6 \mu \mathrm{g}$ / $\mathrm{mL})$ in the absence of serum. The treated cells were fixed for $15 \mathrm{~min}$ in $4 \%$ formaldehyde at room temperature and rinsed three times with PBS for 5 min each. The intracellular distribution of ICG was observed at different time points using an ICG filter equipped fluorescence microscope (BZ-X700, Keyence, Japan). The software of Image $\mathrm{J}$ was used to quantify the mean fluorescence intensity (MFI) of ICG fluorescence from ten random fields of view.

\section{Cell Viability Assay}

Cells were seeded in 24-well plates and allowed to adhere for $24 \mathrm{~h}$. After replacing with serum-free media, the cells were maintained overnight. For cytotoxicity test, RM9 cells were incubated with PLGA-ICG-R848 with different 
concentrations of ICG for $4 \mathrm{~h}$ and irradiated without or with $808 \mathrm{~nm}$ laser $\left(1 \mathrm{~W} / \mathrm{cm}^{2}\right)$ for $10 \mathrm{~min}$. After $24 \mathrm{~h}$, the cell viability was detected using cell proliferation kit (Roche, Germany). For cell proliferation assay, RM9, PC-3, LNCaP and DU-145 cells were firstly treated for 4 $h$ with PBS, PLGA, PLGA-R848, PLGA-ICG and PLGAICG-R848, containing the same concentration of ICG or R848 and next irradiated without or with $808 \mathrm{~nm}$ laser $\left(1 \mathrm{~W} / \mathrm{cm}^{2}\right)$ for $10 \mathrm{~min}$. After $24 \mathrm{~h}$, the cell viability was detected using cell proliferation kit (Roche, Germany).

\section{Generation and Maturation of BMDCs}

Immature BMDCs were generated according to a standard protocol (BMDC isolation protocol, Abcam, USA). Bone marrow cells were isolated from the femurs and tibias of C57BL/6 mice (male, 8-10 weeks old) on day 0. After filtration on a $40 \mu \mathrm{m}$ cell strainer and red blood cell lysis, bone marrow cells were then seeded in 100-mm Ultra-Low Attachment Culture Dish containing 10 mL RPMI-1640 medium supplemented with $10 \% \mathrm{FBS}, 20 \mathrm{ng} / \mathrm{mL}$ GM-CSF and $10 \mathrm{ng} / \mathrm{mL}$ IL-4. The cells were cultured at $37^{\circ} \mathrm{C}$ in an incubator containing $5 \% \mathrm{CO}_{2}$. On day 3 , an additional $10 \mathrm{~mL}$ of RPMI-1640 medium was added, and then half of the culture medium was changed on day 6. During cultivation, the same concentrations of GM-CSF and IL4 were maintained as above.

On day 8 , both the semi-suspended cells and loosely attached cells were gently collected and plated into 6-well plates. The cells were treated for $48 \mathrm{~h}$ with (1) PBS, (2) PLGA, (3) PLGA-ICG, (4) PLGA-R848, (5) PLGA-ICGR848, (6) R848 (5 $\mu \mathrm{g} / \mathrm{mL})$ and (7) LPS $(0.1 \mu \mathrm{g} / \mathrm{mL})$. LPS at $100 \mathrm{ng} / \mathrm{mL}$ was used as the positive control.

On day 10, BMDCs were stained with anti-CD11c PE (BD Pharmingen ${ }^{\mathrm{TM}}$, \#557401), anti-CD86 FITC (BD Pharmingen ${ }^{\mathrm{TM}}$, \#553691) and anti-CD80 APC (BD Pharmingen ${ }^{\mathrm{TM}}$, \#560016), and then analysed by flow cytometry (Miltenyi Biotec, Germany).

\section{PLGA-ICG-R848 Treatment for Subcutaneous Xenograft Model of RM9-Luc PCa Cells}

Six-week-old male C57BL/6J mice were provided by Japan SLC, Inc. (Shizuoka, Japan) and were acclimated for 2 weeks. Animal experimental protocols were approved by the Ethics Review Committee for Animal Experimentation of Okayama University (No. OKU2018003). The welfare and treatment of the laboratory animals followed the Guideline of Animal Experiment in Okayama University. The use of RM9 cell line was approved by the Okayama University Animal Research Committee. RM9 cells were infected with plasmids containing luciferase and named RM9-Luc cells, which was also reported in our previous studies. ${ }^{22,23}$

For the tumor inoculation, RM9-Luc cells $\left(2 \times 10^{5}\right)$ resuspended in $100 \mu \mathrm{L}$ PBS were subcutaneously inoculated into the left flank of each mouse. Ten days later, the tumor volume reached approximately $150 \mathrm{~mm}^{3}$ and the mice were randomized into the following groups: (1) PBS, (2) PBS+laser, (3)PLGA-ICG+laser, (4)PLGA-ICG-R848 +laser. Nanoparticle suspension in $100 \mu \mathrm{L}(1.6 \mathrm{mg} / \mathrm{kg}$ ICG, $0.33 \mathrm{mg} / \mathrm{kg}$ R848 per mouse) was injected intratumorally. According to the results of cellular uptake experiment and cell viability assay, $808 \mathrm{~nm}$ laser $\left(0.8 \mathrm{~W} / \mathrm{cm}^{2}\right)$ was used to irradiate the tumor for $10 \mathrm{~min}$ after 4 $\mathrm{h}$ injection. During laser irradiation, the subcutaneous temperature was monitored at $2 \mathrm{~min}, 4 \mathrm{~min}, 6 \mathrm{~min}, 8$ min and 10 min by FLIR thermal imager (FLIR Systems Inc., USA). The IVIS-200 Imaging System (Xenogen, Alameda, CA, USA) was applied to acquire bioluminescence images and analyze tumor growth.

Two weeks after treatment, spleens were collected to make single cell splenocyte suspensions. Splenocytes were stained with anti-CD3 PE (BD Pharmingen ${ }^{\mathrm{TM}}$, \#553064), anti-NK1.1 FITC (BD Pharmingen ${ }^{\mathrm{TM}}$, \#553164). The MACSQuant Analyzer 10 (Miltenyi Biotec, Germany) was used to detect and analyze the proportion of natural killer (NK) cells.

\section{Statistical Analysis}

Data are presented as mean \pm standard error $($ mean $\pm \mathrm{SD})$ unless otherwise indicated. Statistical analyses were performed using GraphPad Prism 8.3. Statistical significance was determined by analysis of variance (ANOVA). Differences were regarded as significant at $P<0.05$.

\section{Results}

\section{Preparation and Characterization of PLGA-ICG-R848 NPs}

PLGA-ICG-R848 NPs prepared from a double emulsion assay were characterized by DLS device and SEM. Consistent with the nanoparticle size and shape characterization observed by SEM analysis, a mean diameter of $157.7 \mathrm{~nm}$ was determined by DLS device (Figure 1A and B). DLS device also revealed that the PDI of PLGA-ICG-R848 was 
0.071 and the zeta potential was $-35.1 \mathrm{mV}$, indicating a good stability of NPs in water solution. Then the prepared solution was observed under IVIS-200 Imaging System equipped with ICG filter. Fluorescence could be detected in the solution containing ICG ingredient such as free ICG, PLGA-ICG and PLGA-ICG-R848, but was not observed in PLGA, PLGA-R848, PBS solution (Figure 1C). The characteristic absorption peaks of ICG and R848 were identified by DS-11 microvolume spectrophotometer, demonstrating the successful encapsulation of ICG and R848 in the PLGA core (Figure 1D). ICG concentration was calculated by subtracting the residual weight from feeding weight. The encapsulation efficiency and loading capacity were $(65.61 \pm 2.09) \%$ and $(0.82$ $\pm 0.03) \%$, respectively, for ICG, while they were $(8.363$ $\pm 0.325) \%$ and $(0.131 \pm 0.004) \%$, respectively, for R848.

\section{Successful Cellular Uptake of PLGA-ICG- R848 NPs and They Inhibit the Cell Viability of PCa Cells After Laser Irradiation}

To confirm the successful cellular uptake of NPs, RM9 cells were treated with PLGA, PLGA-ICG, PLGA-R848 and PLGA-ICG-R848 for $2 \mathrm{~h}$ or $12 \mathrm{~h}$. After incubation with NPs containing ICG ingredient for $2 \mathrm{~h}$, fluorescence of ICG appeared in RM9 cells, and stronger fluorescence was observed when the incubation time was longer. But the groups without ICG ingredient presented no ICG fluorescence (Figure 2A). As shown in Figure 2B, there was no significant difference in the MFI of ICG fluorescence between PLGA-R848 (2h) and PLGA-ICG-R848 (2h), or between PLGA-R848 (12h) and PLGA-ICG-R848 (12h).

For cytotoxicity test, RM9 cells were incubated with PLGA-ICG-R848 with different concentrations of ICG. The cell viability exhibited no significant difference regardless of different ICG concentrations in the group of PLGA-ICG-R848. But after exposing the treated RM9 cells to $808 \mathrm{~nm}$ laser irradiation, the viability rate significantly decreased in a concentration-dependent manner (Figure 2C). For cell proliferation assay, RM9 cells and human prostate cancer cell lines including PC-3, LNCaP and DU-145 were treated for $4 \mathrm{~h}$ with PBS, PLGA, PLGA-R848, PLGA-ICG and PLGA-ICG-R848 and next irradiated without or with laser. The cell viability of PLGA-ICG+laser and PLGA-ICG-R848+laser was significantly inhibited after $24 \mathrm{~h}$ culture. But no significant inhibition was found in groups of PBS, PBS+laser, PLGA+laser and PLGA-R848+laser (Figure 2D).

\section{PLGA-ICG-R848 NPs Promote BMDCs Maturation}

On day 10 when BMDCs were stimulated for $48 \mathrm{~h}$, more cells with enlarged volume and dendritic structure were observed by microscopy in groups of PLGA-R848, PLGAICG-R848, R848 (5 $\mu \mathrm{g} / \mathrm{mL})$ and LPS $(0.1 \mu \mathrm{g} / \mathrm{mL})$ than in other groups. Both the non-adherent and loosely adherent cells were gently collected, and flow cytometry was performed to detect CD11c marker (a specific surface marker of murine dendritic cells), CD86 and CD80 markers (costimulatory factors to provide the second signal of $\mathrm{T}$ cell activation). The representative results of $\mathrm{CD} 11 \mathrm{c}+\mathrm{CD} 86+$ cells and CD11c+CD80+ cells are shown in Figure 3A and $\mathrm{B}$, respectively. The ratios of $\mathrm{CD} 11 \mathrm{c}+$ cells were more than $91 \%$ in all groups and were not significantly different between any two groups, indicating a relatively high purity of BMDCs.

The ratios of $\mathrm{CD} 11 \mathrm{c}+\mathrm{CD} 86+$ cells were $(38.54$ $\pm 1.30) \%, \quad(41.41 \pm 6.92) \%, \quad(44.04 \pm 3.24) \%, \quad(62.84$ $\pm 1.76) \%, \quad(63.70 \pm 2.08) \%, \quad(65.71 \pm 9.14) \%$ and $(90.96$ $\pm 4.31) \%$, in the groups of Control, PLGA, PLGA-ICG,

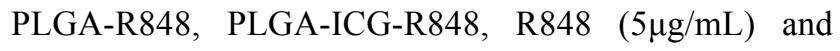
LPS $(0.1 \mu \mathrm{g} / \mathrm{mL})$, respectively (Figure $3 \mathrm{C})$. Similar to the proportional change of $\mathrm{CD} 11 \mathrm{c}+\mathrm{CD} 86+$ cells, the ratios of $\mathrm{CD} 11 \mathrm{c}+\mathrm{CD} 80+$ cells were $(40.43 \pm 1.22) \%$, $(47.16 \pm 6.66) \%, \quad(44.62 \pm 6.23) \%, \quad(68.56 \pm 1.86) \%, \quad(70.61$ $\pm 3.70) \%, \quad(71.54 \pm 3.77) \%$ and $(91.59 \pm 0.57) \%$, in the groups of Control, PLGA, PLGA-ICG, PLGA-R848, PLGA-ICG-R848, R848 $(5 \mu \mathrm{g} / \mathrm{mL})$ and LPS $(0.1 \mu \mathrm{g} /$ $\mathrm{mL}$ ), respectively (Figure $3 \mathrm{C}$ ). The percentage of CD11c+CD86+ cells in any group of PLGA-R848, PLGA-ICG-R848 and R848 $(5 \mu \mathrm{g} / \mathrm{mL})$ was significantly higher than that in any group of Control, PLGA and PLGA-ICG, and it was the same as CD11c+CD80+ cells.

\section{PLGA-ICG-R848 NPs Plus NIR Laser Exhibit the Anti-Tumor Effect on PCa Mouse Model}

To assess the anti-tumor effect of PLGA-ICG-R848 NPs, we established the RM9-Luc subcutaneous tumor xenograft model. During laser irradiation $\left(0.8 \mathrm{~W} / \mathrm{cm}^{2}, 10 \mathrm{~min}\right)$, the subcutaneous temperatures at 2, 4, 6, 8 and $10 \mathrm{~min}$ were controlled around $50^{\circ} \mathrm{C}$ in the groups of PLGA-ICG +laser, PLGA-ICG-R848+laser and below $40^{\circ} \mathrm{C}$ in the group of PBS+laser (Figure 4A and B).

Two weeks after treatment, in vivo bioluminescence imaging was performed to monitor tumor growth and 


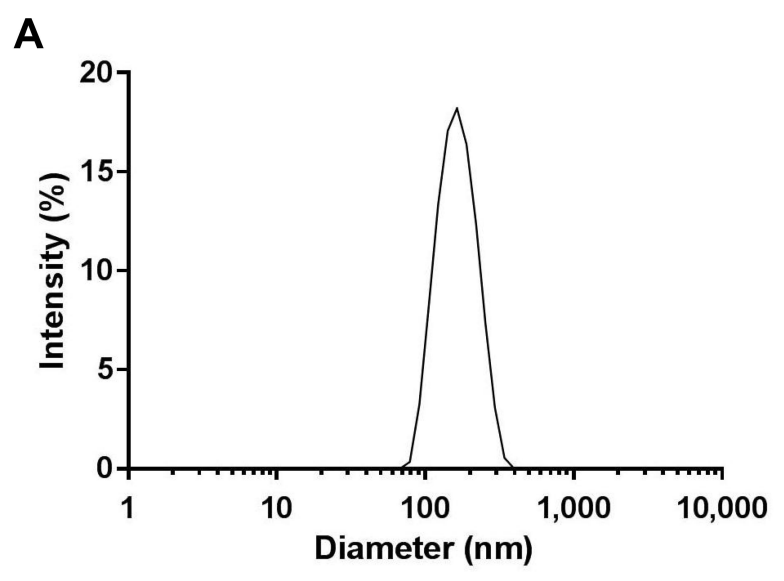

C

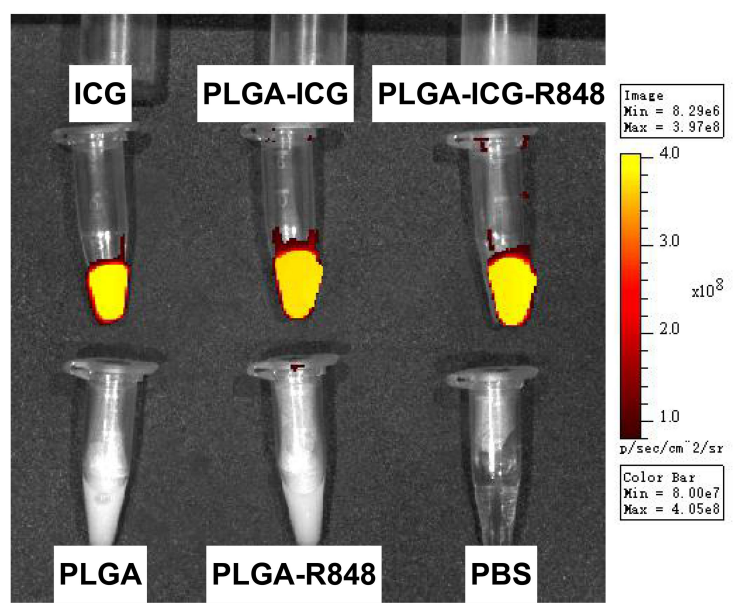

B

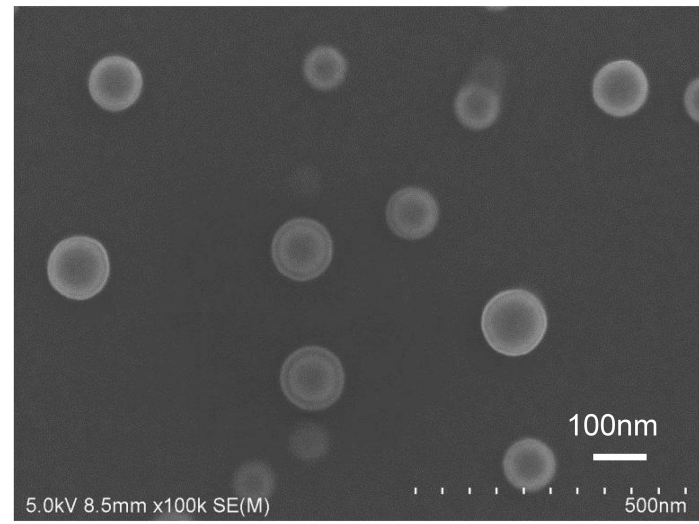

D

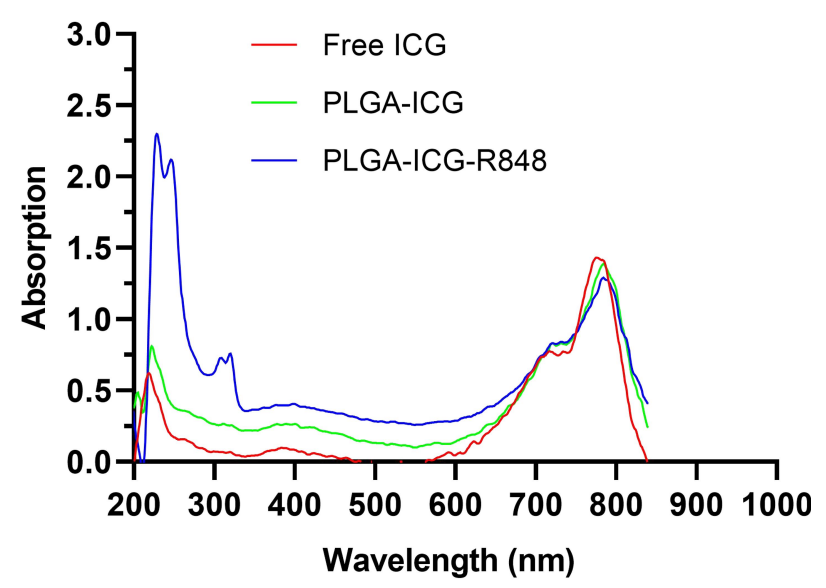

Figure I Preparation and characterization of PLGA-ICG-R848 NPs. (A) The mean diameter of PLGA-ICG-R848 NPs was determined by DLS device. (B) SEM analysis was applied to identify the nanoparticle size and shape characterization. Scale bar $=100 \mathrm{~nm}$. (C) Under IVIS-200 Imaging System, ICG fluorescence could be detected in groups of free ICG, PLGA-ICG and PLGA-ICG-R848, but was not observed in PLGA, PLGA-R848 and PBS. (D) The characteristic absorption peaks of ICG and R848 were identified by DS-II microvolume spectrophotometer, demonstrating the successful encapsulation of ICG and R848 in the PLGA core.

bioluminescent images were quantified using Living Image 3.2 software. The luminescent signals in the groups of (3)PLGA-ICG+laser and (4)PLGA-ICG-R848+laser were significantly lower than those in the groups of (1) PBS and (2) PBS+laser (Figure 4C and D).

To evaluate the immune response after PLGA-ICGR848 treatment, spleens were collected for flow cytometry. In splenocytes, the ratio of CD3-NK1.1+ cells in (4) PLGA-ICG-R848+laser was $(3.96 \pm 1.88) \%$, which was significantly higher than $(0.99 \pm 0.10) \%$ in (1) PBS (Figure 4E and F).

\section{Discussion}

To obtain better efficacy against $\mathrm{PCa}$, a growing interest has been attracted to develop multi-functional nanoparticles, such as chemotherapy, gene therapy and immunotherapy. ${ }^{24-26}$ In this study, we successfully synthesized dual-functional PLGAICG-R848 NPs with three clinically proven ingredients (PLGA, ICG and R848), and demonstrated the in vitro and in vivo anticancer efficacy of PLGA-ICG-R848 NPs when irradiated with $808 \mathrm{~nm}$ NIR laser (Figure 5A). The data of particle size, zeta potential and PDI revealed a good stability of PLGA-ICG-R848 NPs. We measured the mean particle size of $157.7 \mathrm{~nm}(>100 \mathrm{~nm})$. According to the related studies, ${ }^{27,28}$ the mean particle size and our results of cellular uptake experiment, PLGA-ICG-R848 NPs may be internalized mainly by clathrin-mediated endocytic pathway, and the results of cell viability assay indicate that PLGA-ICG-R848 NPs without laser irradiation had a low toxicity to prostate cancer cells. The cellular uptake of NPs containing ICG was demonstrated by our observation of stronger ICG fluorescence in RM9 cells with longer incubation time. 
A

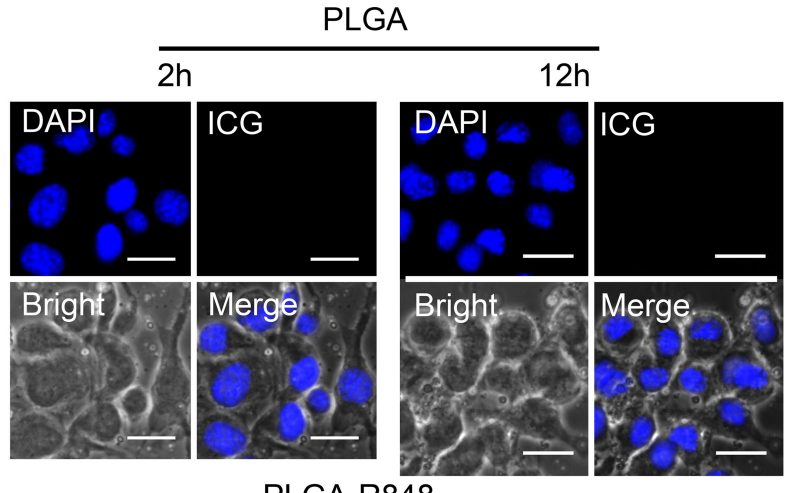

PLGA-R848

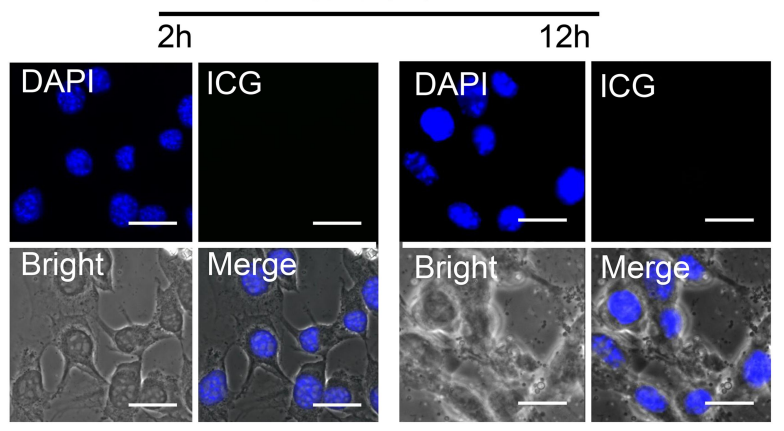

B

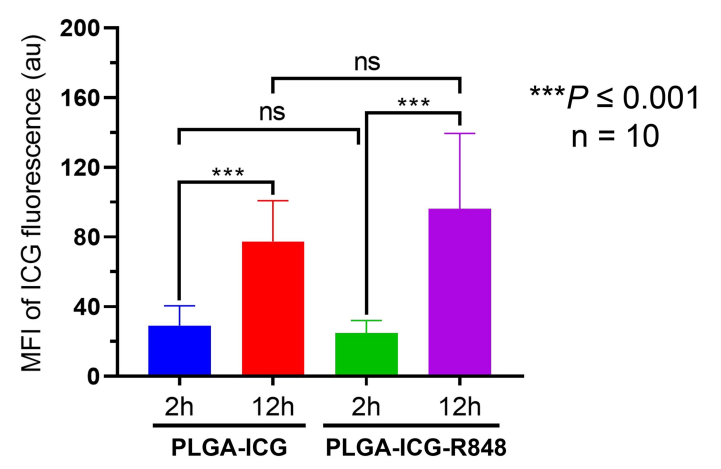

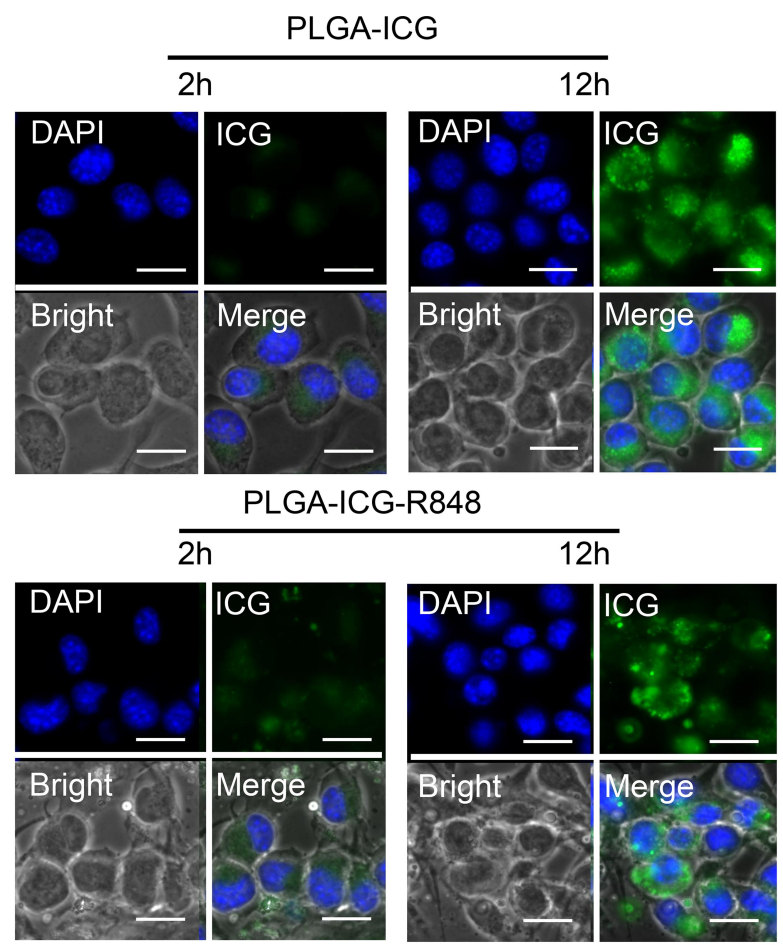

C
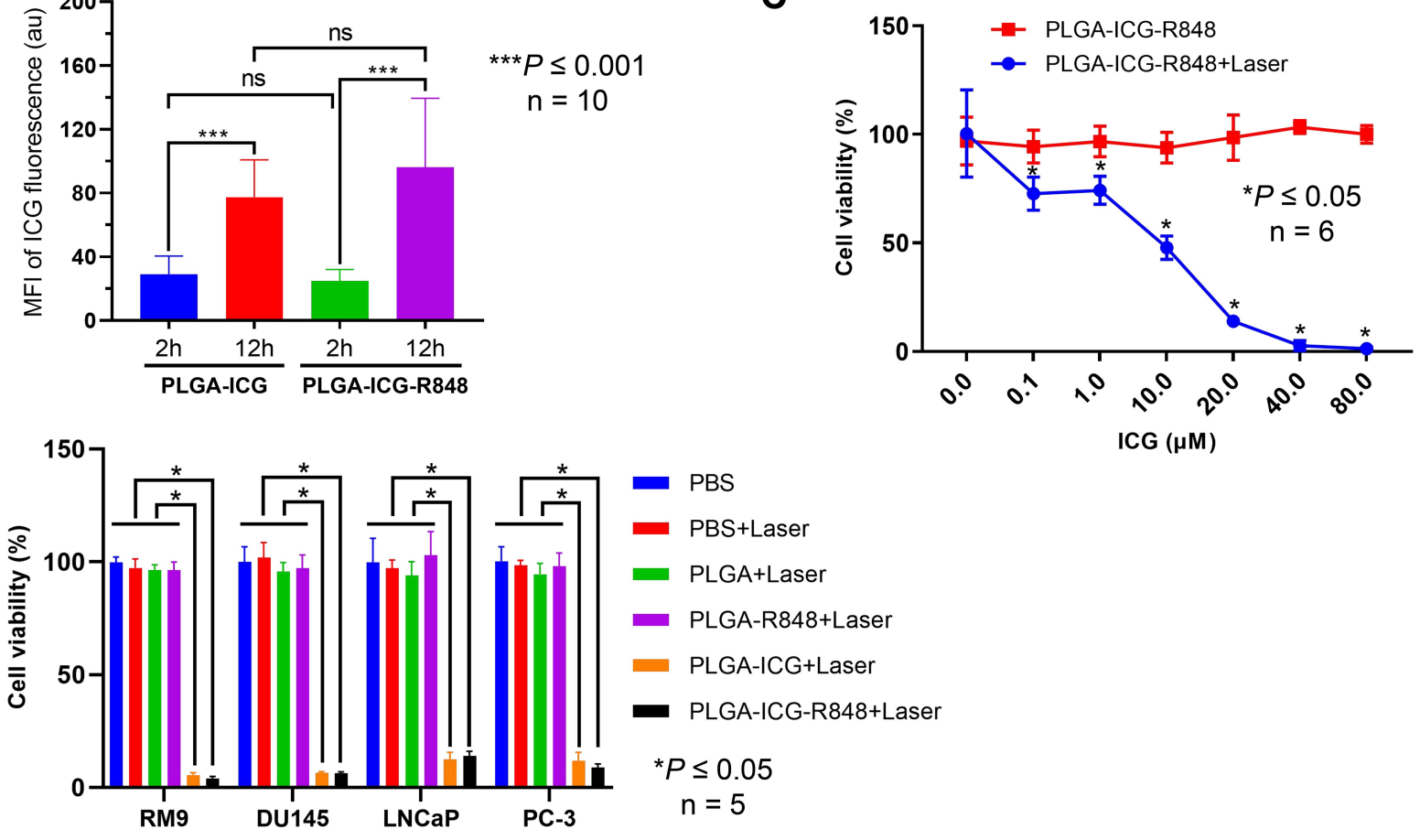

Figure 2 PLGA-ICG-R848 NPs plus laser irradiation inhibited PCa cell viability. (A) ICG fluorescence was observed in RM9 cells after $2 \mathrm{~h}$ treatment, and stronger fluorescence was observed with the longer incubation time in groups of PLGA-ICG and PLGA-ICG-R848. (B) Mean fluorescence intensity (MFI) of ICG fluorescence was quantified in groups of PLGAICG and PLGA-ICG-R848. ( $\left.{ }^{* * *} P<0.001, \mathrm{n}=10\right)$. (C) After RM9 cells were treated with PLGA-ICG-R848 containing different ICG concentrations, the cell viability exhibited no significant difference in the group of PLGA-ICG-R848 without laser irradiation. But the viability rate significantly decreased in a dose-dependent manner in the group of PLGA-ICG-R848 +laser. $(* P<0.05, n=6)$. (D) After $4 \mathrm{~h}$ treatment, prostate cancer cells of RM9, PC-3, LNCaP and DU-I 45 were irradiated without or with $808 \mathrm{~nm}$ laser. The cell viability of PLGA-ICG + laser and PLGA-ICG-R848+laser was significantly inhibited, but there was no significant inhibition in groups of PBS, PBS+laser, PLGA+laser and PLGA-R848+laser ( $* P<0.05, n=5$ ).

A growing number of low-risk or intermediate-risk PCa cases are diagnosed with the advance of screening techniques. ${ }^{29}$ Focal therapy may play an increasingly important role in PCa treatment for its efficient removal of prostate cancer lesions without damaging healthy tissues and nearby organs. ${ }^{16}$ In recent years, PTT activated by NIR light is 
preferred for cancer therapy due to such advantages as easy operation, accurate positioning, noninvasive penetrating tissue to a certain depth and low toxicity. ${ }^{30}$ The FDA-approved NIR cyanine dye, ICG, can not only cause singlet oxygen generation for PDT, but also convert absorbed light energy into heat for PTT. ${ }^{31,32}$ In our study, ICG was encapsulated into PLGA NPs to improve ICG stability and enhance PTT against PCa, which is mainly discussed here. In the cell viability assay, we found PLGA-ICG and PLGA-ICG-R848 under laser irradiation significantly inhibited the cell viability of RM9 cells and human prostate cancer cell lines, but the NIR light itself caused no cytotoxic effect in the absence of photosensitizers including ICG. Accumulating evidence indicates that a temperature of over $43^{\circ} \mathrm{C}$ caused by PTT can exert an irreversible damage to cancer cells. ${ }^{33,34}$ During laser irradiation, the subcutaneous temperatures in the groups of PLGA-ICG+laser and PLGAICG-R848+laser were controlled around $50^{\circ} \mathrm{C}$, which could irreversibly damage PCa cells. Moreover, the groups of PLGA-ICG+laser and PLGA-ICG-R848+laser had lower luminescent signals of RM9-Luc cells than the control groups, revealing the ICG-induced PTT to kill PCa cells. We also detected the residual ICG under IVIS-200 Imaging System at one week and two weeks after treatment with PLGA-ICGR848 NPs plus laser irradiation (data not shown), indicating the sustained release of ICG as well as R848, and a second treatment with laser irradiation for better efficacy.

Although focal therapy is an attractive option for wellselected patients, it should be noted that both uncertain early recurrence risk and a lack of long-term clinical outcome pose obstacles to its routine use recommended by most urologic associations. ${ }^{7}$ Therefore, close monitoring after focal therapy and its combination with systemic therapy are required to ensure a reduced risk of recurrence and the enhanced antiPCa effects. However, there are still few reports studying the synergistic effects of focal therapy combined with immunotherapy against PCa. Previous studies have reported that NIR PTT-based in situ vaccination has the potential to enhance immune response and immunological memory against tumor recurrence and metastasis with lower toxicity but less expensive, less ethic and safety concerns than conventional cancer vaccines, even for such immunologically cold tumors as breast cancer. ${ }^{35-38}$ Similarly, intratumoral injection of PLGA-ICG-R848 followed by $808 \mathrm{~nm}$ laser ablation promoted the release of TAAs in situ to trigger specific immune response against $\mathrm{PCa}$ and the release of R848 as an immunopotentiator to enhance the immune response of in situ vaccination and produce longer-lasting immunity. Chen et al reported that PLGA NPs co-loaded with ICG and imiquimod (R837, TLR7 agonist) using o/w single-emulsion method contribute to an elevated secretion of such pro-inflammatory cytokines as interleukin-6 (IL-6), tumor necrosis factor alpha (TNF- $\alpha$ ) and interleukin-12p70 (IL-12p70) by mature DCs in mice bearing either $4 \mathrm{~T} 1$ or CT26 tumors. ${ }^{37}$ The immune adjuvant R848 used in our study has been reported to enhance antitumor immunity in multiple cancers by the differentiation of myeloid-derived suppressor cells (MDSCs) into M1-like macrophages, ${ }^{39}$ the polarization of macrophages, ${ }^{40}$ the maturation of DCs, ${ }^{41}$ and the elevated secretion of pro-inflammatory cytokines. Interestingly, according to the recent findings by Kim et al, mature DCs stimulated by TLR7/8-agonist-loaded NPs increase the secretion of pro-inflammatory cytokines and the expression of costimulatory molecules, both of which can promote splenic NK cell activation. ${ }^{42}$ There was no significant difference in promoting BMDCs maturation between free R848 and PLGA-ICG-R848 NPs. For PLGAICG-R848 NPs, the release of R848 at $48 \mathrm{~h}$ was demonstrated to cause a similar result of BMDCs maturation compared with free R848. However, compared with free R848, R848 encapsulated in PLGA NPs has been proven to augment the immune response and exhibit strong local immune activation without inducing systemic cytokine release and immune tolerance. ${ }^{13,43}$ Correspondingly, PLGA-ICG-R848 NPs treatment was found to increase the maturation markers of BMDCs and contribute to an elevated ratio of splenic NK cells in mice bearing RM9-Luc tumor (Figure 5B). NK cells may kill RM9 PCa cells through the release of such cytotoxic molecules as perforin and granzyme, and the secretion of cytokines such as interferon-gamma (IFN- $\gamma$ ) to promote the differentiation of T helper type 1 (Th1) cells. ${ }^{44,45}$ Compared with a single injection of R848, cycles of repeated injections were developed to circumvent immune tolerance and improve the efficacy of cancer immunotherapy. ${ }^{46}$ To avoid repeated injections, R848 encapsulated in NPs can slowly release, and subsequently reduce potential adverse events and safety risks initiated by cytokine storm. ${ }^{40-42}$ To date, although a growing number of R848 preclinical and clinical trials have been performed to verify its efficacy on multiple cancers, there are a lack of studies about PCa treatment. Recently, Islam et al demonstrated that mRNA vaccine pulsed with R848 adjuvant exhibits the anti-tumor efficacy in syngeneic mouse models of prostate cancer. ${ }^{47}$ Our data prove that PLGA-ICG-R848 induced the significant maturation of BMDCs, and its combination with laser ablation increased the proportion of splenic NK cells, which may further contribute to a stronger anticancer efficacy against 
A

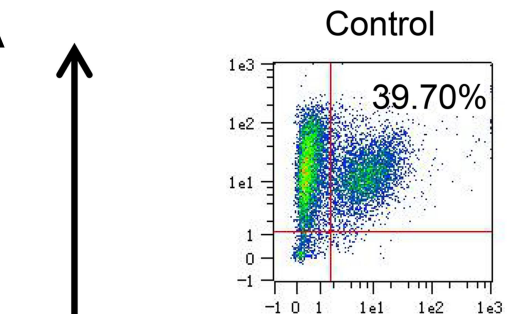

PLGA

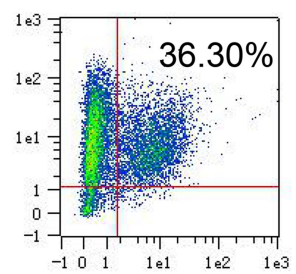

PLGA-ICG

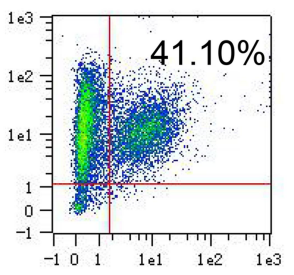

PLGA-R848 PLGA-ICG-R848
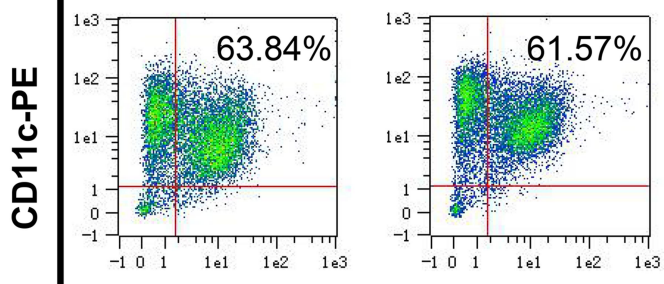

$\mathrm{R} 848(5 \mu \mathrm{g} / \mathrm{mL})$

LPS $(0.1 \mu \mathrm{g} / \mathrm{mL})$
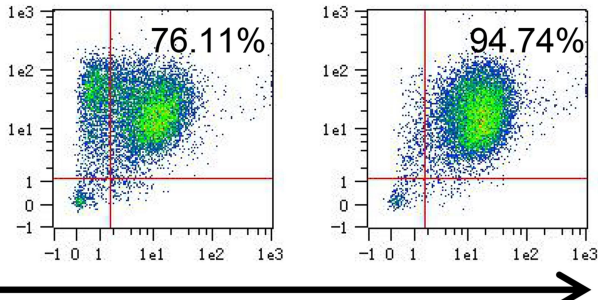

CD86-FITC

B
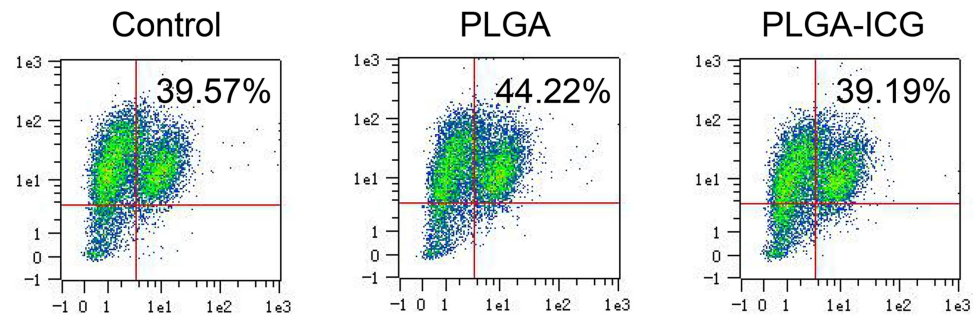

PLGA-R848

PLGA-ICG-R848
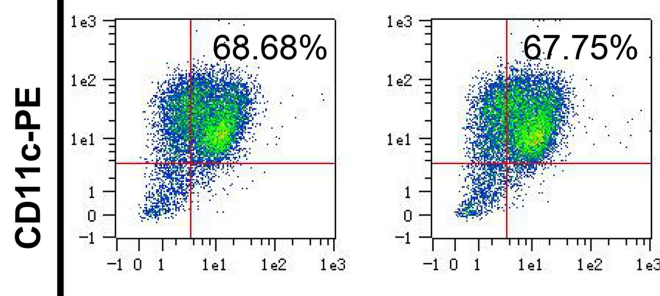

R848 $(5 \mu \mathrm{g} / \mathrm{mL})$

LPS $(0.1 \mu \mathrm{g} / \mathrm{mL})$
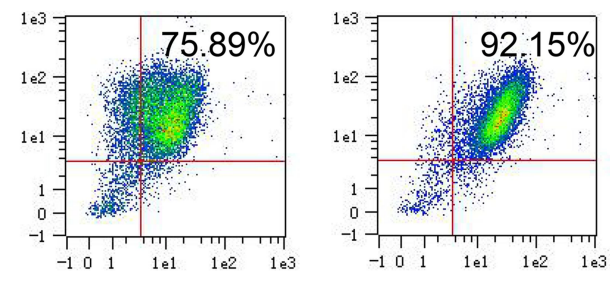

CD80-APC

C

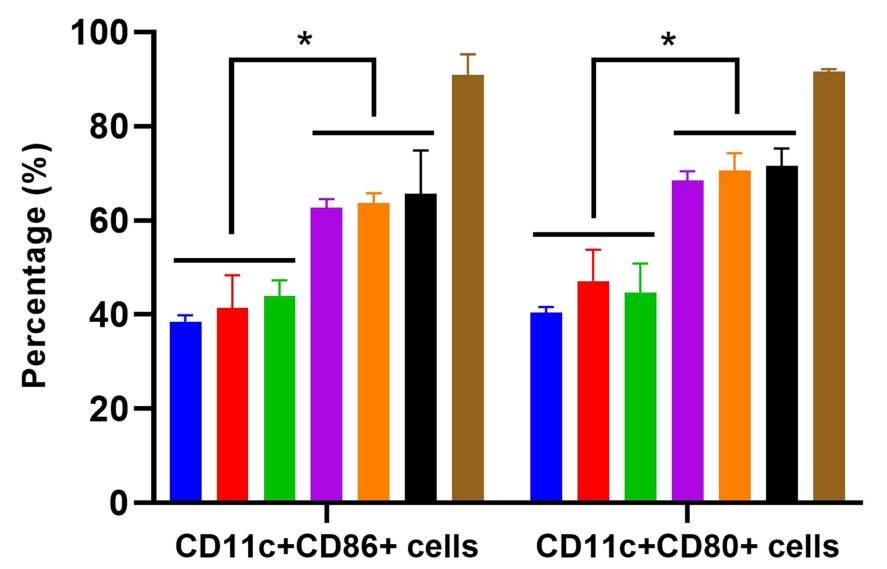

$\square$

(2) PLGA

(3) PLGA-ICG

(4) PLGA-R848

(5) PLGA-ICG-R848

(6) R848

(7) LPS

${ }^{*} P \leq 0.05$

$\mathrm{n}=3$

Figure 3 PLGA-ICG-R848 increases the maturation markers of BMDCs. (A and B) On day 8, the BMDCs were collected and then treated with (I) PBS, (2) PLGA, (3) PLGA-ICG, (4) PLGA-R848, (5) PLGA-ICG-R848, (6) R848 (5 $\mu \mathrm{g} / \mathrm{mL})$ and (7) LPS $(0.1 \mu \mathrm{g} / \mathrm{mL})$. After 48h treatment, flow cytometry was performed to analyze the

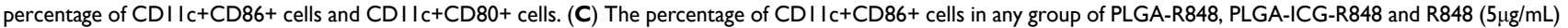
was significantly higher than that in any group of Control, PLGA and PLGA-ICG, and it was the same as CDI Ic $+C D 80+$ cells $(* P<0.05, n=3)$. Data are expressed as the mean \pm SD from three independent experiments. 
A

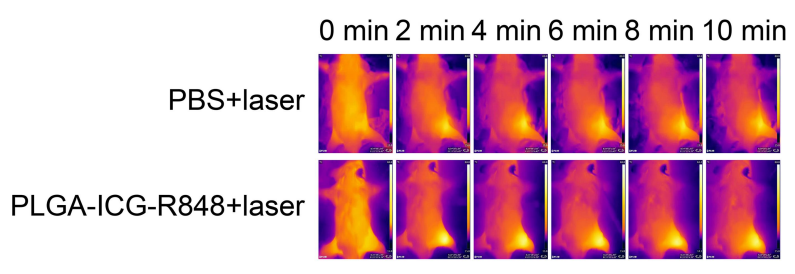

C

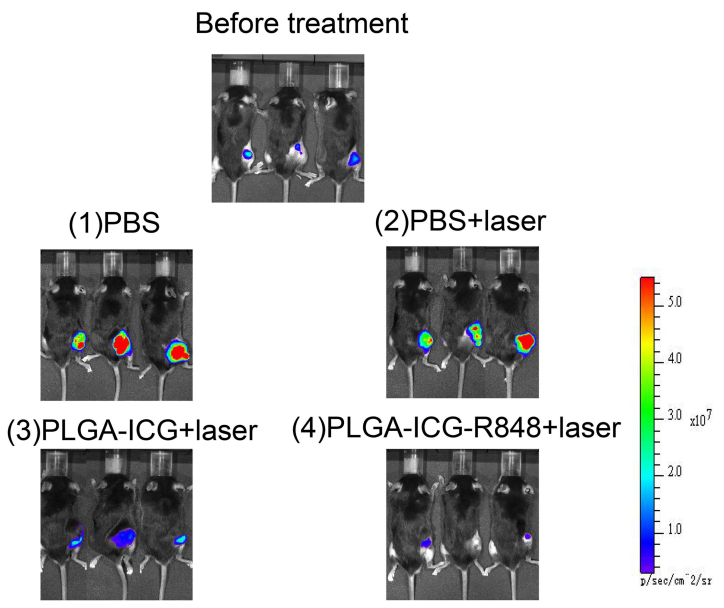

E

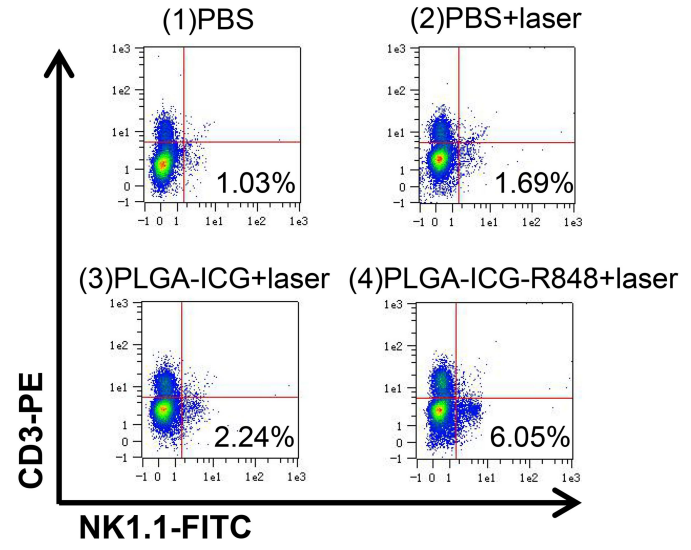

B

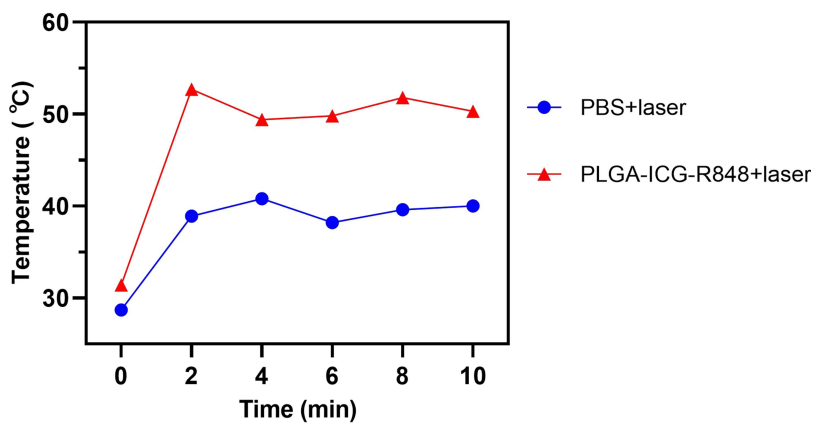

D

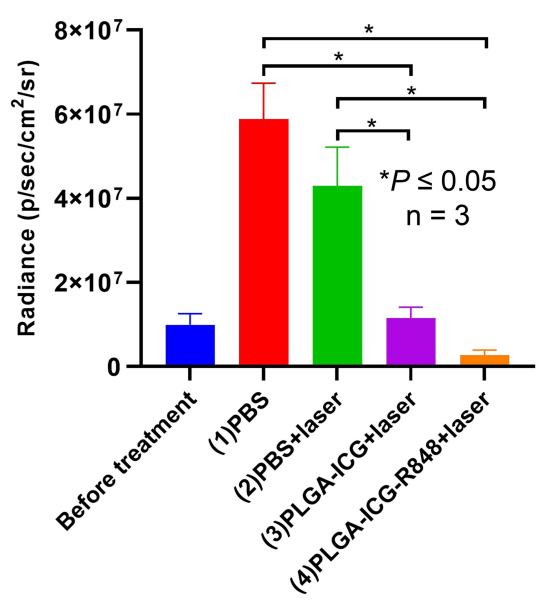

$\mathbf{F}$

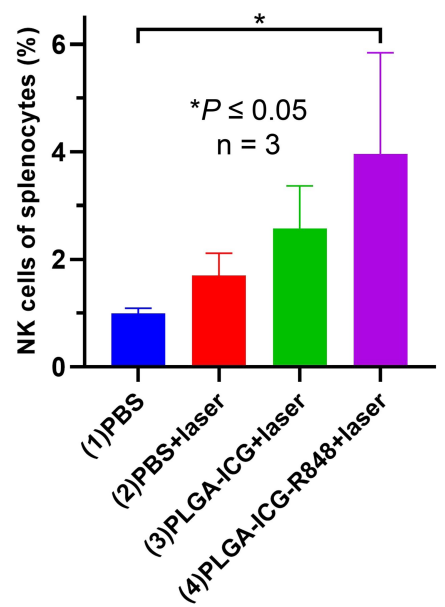

Figure 4 Anti-tumor effect of PLGA-ICG-R848 NPs plus NIR laser in vivo. (A and B) During laser irradiation, the subcutaneous temperatures in groups of PBS+laser and PLGA-ICG-R848+laser were monitored at $2 \mathrm{~min}, 4 \mathrm{~min}, 6 \mathrm{~min}, 8 \mathrm{~min}$ and $10 \mathrm{~min}$ by FLIR thermal imager. (C) Two weeks after photothermal therapy, in vivo bioluminescence imaging was performed to monitor tumor growth and (D) bioluminescent images were quantified $(* P<0.05, n=3)$. (E and $\mathbf{F})$ Spleens were collected after two-week treatment and flow cytometry was used to detect the proportion of NK cells in splenocytes $(* P<0.05, n=3)$.

PCa. Further studies to evaluate the anti-metastasis effect and long-term maintenance of immunological memory against $\mathrm{PCa}$ are also required to better understand the therapeutic potential of PLGA-ICG-R848 NPs with NIR laser.
This is the first report to assess the antineoplastic effect of PLGA-ICG-R848 NPs with NIR laser on a mouse subcutaneous PCa model. However, this model could not well mimic the cancer cells growing 
A

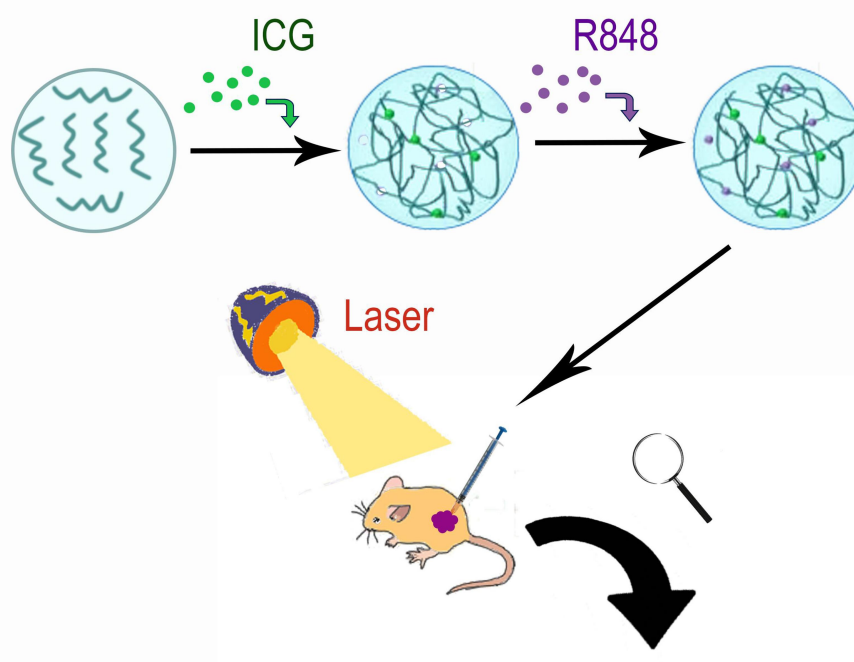

$\bar{B}$

Spleen

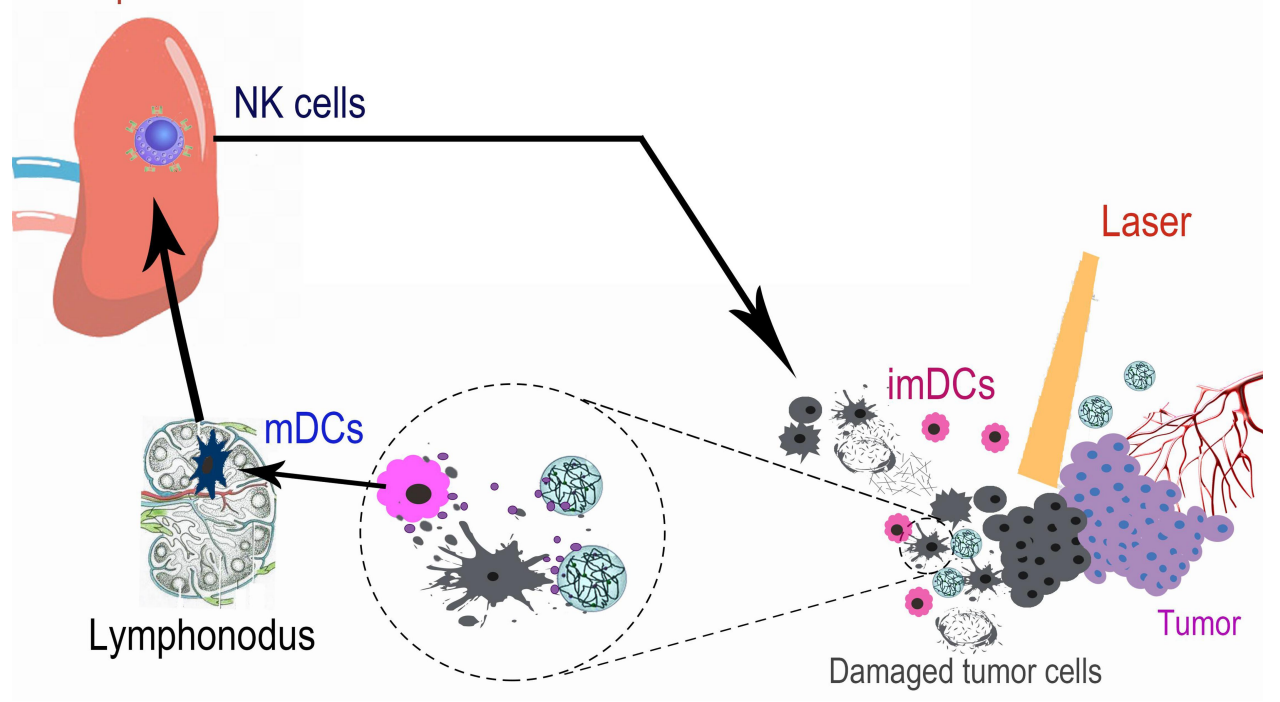

Figure 5 The mechanism of anti-tumor efficacy induced by PLGA-ICG-R848 NPs plus NIR laser. (A) The preparation of PLGA-ICG-R848 NPs and their application in vivo. (B) Schematic illustration of PLGA-ICG-R848 NPs for PTT and anti-tumor immune response against PCa.

Abbreviations: NK cells, natural killer cells; $\mathrm{mDC}$, mature dendritic cells; imDCs, immature dendritic cells.

in their natural location, so the orthotopic mouse model of $\mathrm{PCa}$ should be introduced for future research. Meanwhile, multiparametric magnetic resonance imaging (mpMRI) can provide anatomical, functional, and physiological parameters of prostate diseases, ${ }^{48}$ which will be of great help to real-time localization and intratumoral injection of PLGA-ICG-R848 NPs when combining PTT with immunotherapy against $\mathrm{PCa} .{ }^{49}$

\section{Conclusion}

Here we reported a dual-functional PLGA-ICG-R848 NPs which combined PTT with immunotherapy against PCa.
PLGA-ICG-R848 NPs under NIR laser irradiation significantly inhibited the growth of human and mouse prostate cancer cell lines. Furthermore, PLGA-ICG-R848 NPs could significantly increase the maturation markers of BMDCs. In the subcutaneous xenograft model of mouse PCa cell line, both the anti-tumor effect and immune response were enhanced by PLGA-ICG-R848 NPs-based laser irradiation. To sum up, the dual-functional PLGA-ICG-R848 NPs under NIR laser irradiation exhibit the anti-tumor efficacy for $\mathrm{PCa}$ treatment in vitro and in vivo. Therefore, these findings could have significant implications in the development of therapeutic strategies against low- or intermediate-risk PCa by combining PTT with immunotherapy. 


\section{Abbreviations}

PCa, prostate cancer; NPs, nanoparticles; PTT, photothermal therapy; PLGA, poly(lactic-co-glycolic acid); ICG, indocyanine green; TLR7/8, toll-like receptor 7/8; BMDCs, bone marrow-derived dendritic cells; TME, tumor microenvironment; TAAs, tumor-associated antigens; FDA, Food and Drug Administration; R848, resiquimod; HIFU, high-intensity focused ultrasound; PDT, photodynamic therapy; PSA, Prostate Specific Antigen; NIR, near-infrared; ROS, reactive oxygen species; w/o/w, water-in-oil-in-water; DLS, dynamic light scattering; PDI, polydispersity index; EE\%, encapsulation efficiency; LC $\%$, loading capacity; SEM, scanning electron microscope; HPLC, high performance liquid chromatography; NK cells, natural killer cells; SD, standard error; ANOVA, analysis of variance; IL-6, interleukin-6; TNF- $\alpha$, tumor necrosis factor alpha; IL-12p70, interleukin-12p70; MDSCs, myeloid-derived suppressor cells; IFN- $\gamma$, interferon-gamma; Th1 cells, T helper type 1 cells; mpMRI, multiparametric magnetic resonance imaging; mDCs, mature dendritic cells; imDCs, immature dendritic cells.

\section{Acknowledgments}

This research was funded by the Ministry of Education, Culture, Sports, Science and Technology of Japan (grant No. 17K11138), Kobayashi Foundation, and the China Scholarship Council. We thank Xianwen Tan, Hideo Ueki and Shunai Li at Okayama University for their technical guidance. Wenfeng Lin and Chaoming Li are co-first authors for this study.

\section{Disclosure}

Prof. Dr. Yasutomo Nasu reports a licensed patent for $R E I C$ gene. The authors report no other conflicts of interest in this work.

\section{References}

1. Rawla P. Epidemiology of prostate cancer. World J Oncol. 2019;10 (2):63-89. doi:10.14740/wjon1191

2. Siegel RL, Miller KD, Jemal A. Cancer statistics, 2020. CA Cancer $J$ Clin. 2020;70(1):7-30. doi:10.3322/caac.21590

3. Mottet N, van den Bergh RCN, Briers E, et al. EAU-EANM-ESTROESUR-SIOG guidelines on prostate cancer-2020 update. Part 1: screening, diagnosis, and local treatment with curative intent. Eur Urol. 2020

4. Cornford P, van den Bergh RCN, Briers E, et al. EAU-EANM-ESTRO -ESUR-SIOG guidelines on prostate cancer. Part II-2020 update: treatment of relapsing and metastatic prostate cancer. Eur Urol. 2020.
5. Xu J, Qiu Y. Current opinion and mechanistic interpretation of combination therapy for castration-resistant prostate cancer. Asian J Androl. 2019;21(3):270-278. doi:10.4103/aja.aja 1019

6. Boettcher AN, Usman A, Morgans A, VanderWeele DJ, Sosman J, Wu JD. Past, current, and future of immunotherapies for prostate cancer. Front Oncol. 2019;9:884. doi:10.3389/ fonc. 2019.00884

7. Ahdoot M, Lebastchi AH, Turkbey B, Wood B, Pinto PA. Contemporary treatments in prostate cancer focal therapy. Curr Opin Oncol. 2019;31 (3):200-206. doi:10.1097/CCO.0000000000000515

8. Vitkin N, Nersesian S, Siemens DR, Koti M. The tumor immune contexture of prostate cancer. Front Immunol. 2019;10:603. doi:10.3389/fimmu.2019.00603

9. Pan C, Liu H, Robins E, et al. Next-generation immuno-oncology agents: current momentum shifts in cancer immunotherapy. J Hematol Oncol. 2020;13(1):29. doi:10.1186/s13045-020-00862-w

10. Bouzid R, Peppelenbosch M, Buschow SI. Opportunities for conventional and in situ cancer vaccine strategies and combination with immunotherapy for gastrointestinal cancers, a review. Cancers (Basel). 2020;12(5):1121. doi:10.3390/cancers12051121

11. Hammerich L, Bhardwaj N, Kohrt HE, Brody JD. In situ vaccination for the treatment of cancer. Immunotherapy. 2016;8(3):315-330. doi: $10.2217 / \mathrm{imt} .15 .120$

12. Kieffer ME, Patel AM, Hollingsworth SA, Seganish WM. Small molecule agonists of toll-like receptors 7 and 8: a patent review 2014-2020. Expert Opin Ther Pat. 2020;30(11):825-845. doi:10.1080/13543776.2020.1825687

13. Ilyinskii PO, Roy CJ, O'Neil CP, et al. Adjuvant-carrying synthetic vaccine particles augment the immune response to encapsulated antigen and exhibit strong local immune activation without inducing systemic cytokine release. Vaccine. 2014;32(24):2882-2895. doi:10.1016/j.vaccine.2014.02.027

14. Pesapane F, Patella F, Fumarola EM, et al. The prostate cancer focal therapy. Gland Surg. 2018;7(2):89-102. doi:10.21037/gs.2017.11.08

15. Catalona WJ. Prostate cancer screening. Med Clin North Am. 2018;102(2):199-214. doi:10.1016/j.mcna.2017.11.001

16. Lepor H, Gold S, Wysock J. Focal ablation of prostate cancer. Rev Urol. 2018;20(4):145-157. doi:10.3909/riu0809

17. Reinhart MB, Huntington CR, Blair LJ, Heniford BT, Augenstein VA. Indocyanine green: historical context, current applications, and future considerations. Surg Innov. 2016;23(2):166-175. doi:10.1177/1553350615604053

18. Wang $\mathrm{H}$, Li X, Tse BW, et al. Indocyanine green-incorporating nanoparticles for cancer theranostics. Theranostics. 2018;8 (5):1227-1242. doi:10.7150/thno.22872

19. Essa D, Kondiah PPD, Choonara YE, Pillay V. The design of poly (lactide-co-glycolide) nanocarriers for medical applications. Front Bioeng Biotechnol. 2020;8:48. doi:10.3389/fbioe.2020.00048

20. Kim YS, Park JS, Park M, et al. PLGA nanoparticles with multiple modes are a biologically safe nanocarrier for mammalian development and their offspring. Biomaterials. 2018;183:43-53. doi:10.1016/ j.biomaterials.2018.08.042

21. Iqbal M, Zafar N, Fessi H, Elaissari A. Double emulsion solvent evaporation techniques used for drug encapsulation. Int $J$ Pharm. 2015;496(2):173-190. doi:10.1016/j.ijpharm.2015.10.057

22. Xu N, Huang L, Li X, et al. The novel combination of nitroxoline and PD-1 blockade, exerts a potent antitumor effect in a mouse model of prostate cancer. Int J Biol Sci. 2019;15(5):919-928. doi:10.7150/ ijbs. 32259

23. Xu P, Xu N, Guo K, et al. Real-time monitoring of tumor progression and drug responses in a preclinical mouse model of prostate cancer. Oncotarget. 2016;7(22):33025-33034. doi:10.18632/ oncotarget. 8846 
24. Ashrafizadeh M, Hushmandi K, Rahmani Moghadam E, et al. Progress in delivery of siRNA-based therapeutics employing nano-vehicles for treatment of prostate cancer. Bioengineering. 2020;7(3). doi:10.3390/bioengineering7030091.

25. Ashrafizadeh M, Zarrabi A, Hushmandi K, et al. Progress in natural compounds/siRNA co-delivery employing nanovehicles for cancer therapy. ACS Comb Sci. 2020;22(12):669-700. doi:10.1021/ acscombsci.0c00099

26. Zhang J, Wang L, You X, Xian T, Wu J, Pang J. Nanoparticle therapy for prostate cancer: overview and perspectives. Curr Top Med Chem. 2019;19(1):57-73. doi:10.2174/1568026619666190125145836

27. Foroozandeh P, Aziz AA. Insight into cellular uptake and intracellular trafficking of nanoparticles. Nanoscale Res Lett. 2018;13(1):339. doi:10.1186/s11671-018-2728-6

28. Murugan K, Choonara YE, Kumar P, Bijukumar D, Du Toit LC, Pillay V. Parameters and characteristics governing cellular internalization and trans-barrier trafficking of nanostructures. Int J Nanomedicine. 2015;10:2191-2206. doi:10.2147/IJN.S75615

29. Klotz L. Contemporary approach to active surveillance for favorable risk prostate cancer. Asian J Urol. 2019;6(2):146-152. doi:10.1016/j. ajur.2018.12.003

30. Liu B, Li C, Cheng Z, Hou Z, Huang S, Lin J. Functional nanomaterials for near-infrared-triggered cancer therapy. Biomater Sci. 2016;4(6):890-909. doi:10.1039/C6BM00076B

31. Zhou Y, Liu S, Hu C, Cai L, Pang M. A covalent organic framework as a nanocarrier for synergistic phototherapy and immunotherapy. J Mater Chem B. 2020;8(25):5451-5459. doi:10.1039/D0TB00679C

32. Xu P, Liang F. Nanomaterial-based tumor photothermal immunotherapy. Int J Nanomedicine. 2020;15:9159-9180. doi:10.2147/IJN.S249252

33. Nomura S, Morimoto Y, Tsujimoto H, et al. Highly reliable, targeted photothermal cancer therapy combined with thermal dosimetry using a near-infrared absorbent. Sci Rep. 2020;10(1):9765. doi:10.1038/ s41598-020-66646-x

34. Zheng M, Yue C, Ma Y, et al. Single-step assembly of DOX/ICG loaded lipid-polymer nanoparticles for highly effective chemophotothermal combination therapy. ACS Nano. 2013;7 (3):2056-2067. doi:10.1021/nn400334y

35. Zou L, Wang H, He B, et al. Current approaches of photothermal therapy in treating cancer metastasis with nanotherapeutics. Theranostics. 2016;6(6):762-772. doi:10.7150/thno.14988

36. Xu X, Lu H, Lee R. Near infrared light triggered photo/immuno-therapy toward cancers. Front Bioeng Biotechnol. 2020;8:488. doi:10.3389/fbioe.2020.00488

37. Chen Q, Xu L, Liang C, Wang C, Peng R, Liu Z. Photothermal therapy with immune-adjuvant nanoparticles together with checkpoint blockade for effective cancer immunotherapy. Nat Commun. 2016;7(1):13193. doi:10.1038/ncomms13193
38. Sadeghi I, Byrne J, Shakur R, Langer R. Engineered drug delivery devices to address global health challenges. J Control Release. 2021;331:503-514. doi:10.1016/j.jconrel.2021.01.035

39. Liu Z, Xie Y, Xiong Y, et al. TLR 7/8 agonist reverses oxaliplatin resistance in colorectal cancer via directing the myeloid-derived suppressor cells to tumoricidal M1-macrophages. Cancer Lett. 2020;469:173-185. doi:10.1016/j.canlet.2019.10.020

40. Rodell CB, Arlauckas SP, Cuccarese MF, et al. TLR7/8-agonistloaded nanoparticles promote the polarization of tumour-associated macrophages to enhance cancer immunotherapy. Nat Biomed Eng. 2018;2(8):578-588. doi:10.1038/s41551-018-0236-8

41. Chen PM, Pan WY, Wu CY, et al. Modulation of tumor microenvironment using a TLR-7/8 agonist-loaded nanoparticle system that exerts low-temperature hyperthermia and immunotherapy for in situ cancer vaccination. Biomaterials. 2020;230:119629. doi:10.1016/j. biomaterials.2019.119629

42. Kim H, Khanna V, Kucaba TA, et al. TLR7/8 agonist-loaded nanoparticles augment NK cell-mediated antibody-based cancer immunotherapy. Mol Pharm. 2020;17(6):2109-2124. doi:10.1021/ acs.molpharmaceut.0c00271

43. Kim H, Griffith TS, Panyam J. Poly(d,l-lactide-co-glycolide) nanoparticles as delivery platforms for TLR7/8 agonist-based cancer vaccine. $J$ Pharmacol Exp Ther. 2019;370(3):715-724. doi:10.1124/ jpet.118.254953

44. Ferlazzo G, Morandi B. Cross-talks between natural killer cells and distinct subsets of dendritic cells. Front Immunol. 2014;5:159. doi:10.3389/fimmu.2014.00159

45. Bottcher JP, Bonavita E, Chakravarty P, et al. NK cells stimulate recruitment of $\mathrm{CDC} 1$ into the tumor microenvironment promoting cancer immune control. Cell. 2018;172(5):1022-1037e1014. doi:10.1016/j.cell.2018.01.004

46. Michaelis KA, Norgard MA, Levasseur PR, et al. Persistent toll-like receptor 7 stimulation induces behavioral and molecular innate immune tolerance. Brain Behav Immun. 2019;82:338-353. doi:10.1016/j.bbi.2019.09.004

47. Islam MA, Rice J, Reesor E, et al. Adjuvant-pulsed mRNA vaccine nanoparticle for immunoprophylactic and therapeutic tumor suppression in mice. Biomaterials. 2021;266:120431. doi:10.1016/j. biomaterials.2020.120431

48. Girometti R, Cereser L, Bonato F, Zuiani C. Evolution of prostate MRI: from multiparametric standard to less-is-better and different-is better strategies. Eur Radiol Exp. 2019;3(1):5. doi:10.1186/s41747019-0088-3

49. Connor MJ, Gorin MA, Ahmed HU, Nigam R. Focal therapy for localized prostate cancer in the era of routine multi-parametric MRI. Prostate Cancer Prostatic Dis. 2020;23(2):232-243. doi:10.1038/ s41391-020-0206-6
International Journal of Nanomedicine

\section{Publish your work in this journal}

The International Journal of Nanomedicine is an international, peerreviewed journal focusing on the application of nanotechnology in diagnostics, therapeutics, and drug delivery systems throughout the biomedical field. This journal is indexed on PubMed Central, MedLine, CAS, SciSearch ${ }^{\mathbb{B}}$, Current Contents ${ }^{\mathbb{B}} /$ Clinical Medicine, $^{2}$
Journal Citation Reports/Science Edition, EMBase, Scopus and the Elsevier Bibliographic databases. The manuscript management system is completely online and includes a very quick and fair peer-review system, which is all easy to use. Visit http://www.dovepress.com/ testimonials.php to read real quotes from published authors. 